\title{
Search for short-time phase effects in the electronic damage evolution \\ - a case study with silicon
}

G. Schiwietz, K. Czerski ${ }^{+}$, R. Hellhammer, M. Roth, F. Staufenbiel ${ }^{++}$ Hahn-Meitner-Institut, Bereich SF, Glienicker Str. 100, 14109 Berlin, Germany

R.C. Fadanelli, and P.L. Grande

Instituto de Física da Universidade Federal do Rio Grande do Sul, Avenida Bento Gonçalves 9500, 91501 - 970, Porto Alegre, RS, Brazil

This work focusses on the production and decay properties of inner-shell vacancies and valence-band excitations induced by swift highly charged ions interacting with amorphous and crystalline Si. High resolution electron spectra have been taken for fast heavy ions at 1.78 to $5 \mathrm{MeV} / \mathrm{u}$ as well as for electrons of similar velocity incident on atomically clean Si targets of well defined phase. Various Auger-electron structures are analyzed concerning their width, their intensity and exact peak position. All measured peaks show a small shift towards lower energy when the charge of the projectile is increased. This finding is an indication for a nuclear-track potential inside the ion track. A detailed analysis of the Auger-electron spectra for amorphous Si and crystalline $\operatorname{Si}(111) 7 \times 7$ points to a small but significant phase effect in the short-time dynamics of ion tracks.

PACS: 79.20.Rf,79.20.Fv,72.20.Jv,32.80.Hd,31.70.Hq,72.15.Lh 


\section{Introduction}

High-resolution ion-beam techniques and especially swift heavyion based analytical techniques are often very sensitive to electronic damage. This is due to the fact that the high kinetic energy of fast ions is transferred virtually only to the electrons of the medium [1]. Charged regions [2,3] as well as hot electrons [4-6] on a nanoscopic scale trigger the subsequent atomic motion and rearrangement inside ion tracks [3,7]. The most important scenarios for track effects with energy-density thresholds are Coulomb explosion [2] due to the mutual repulsion of ionized target atoms, spontaneous lattice relaxation due to long lived repulsive states [4] and the electronic thermal spike due to electron-phonon coupling [5] or individual electron-ion collisions [6]. A critical survey of the electronic thermal-spike and related models may be found in [8]. All of the above mechanisms may finally yield an unordered atomic motion and if a critical local lattice temperature is exceeded, permanent atomic rearrangement may result on a time scale of 0.1 to $10 \mathrm{ps}$. Amorphous materials are typically subject to reduced energy-loss thresholds for electronically driven modifications. Of course the solid-state phase itself should have an influence on the atomic motion and correspondingly there might an influence on damage creation as well.

The solid-state phase, however, has also an influence on the initial excitations and especially on the electronic transport properties via the existence of electronic trap states, as well as a modification of electronic gaps and other band-structure effects. Using amorphous and crystalline $\mathrm{Si}$ as a test system for the investigation of electronic effects, we may apply in-situ high-resolution Auger spectroscopy to investigate track-related properties. This material is not only important for practical purposes, but both phases are also known to be insensitive to electronic damage pro- 
duction. This stability of Si simplifies our on-line measurements and interpretations very much. Results from the corresponding surface-sensitive experiments, performed under ultra-high vacuum conditions, are presented and discussed, considering their impact on materials-modification processes.

In previous investigations, it was possible to distinguish between the appearance of a nuclear-track potential in some polymers [9-12], related to Coulomb explosion, and also high electron temperatures in semi-metals (graphite and graphite-like amorphous carbon) [12-14], metals (Al, Be) and some metallic glasses [15]. These quantities are related to the energetic positions and widths of Auger lines that yield different snapshots of the short-time evolution, some $10^{-15} \mathrm{~s}$ after the passage of heavy ions. The electron temperature influences the populated electronic density-of-states and hence, the initial Auger-electron source-spectra [12-14,16]. The electron temperature leads also to an enhanced absorption of Auger electrons during their transport to the surface and modifies the corresponding Auger angular distribution [17]. The nucleartrack potential resulting from ionization and charge separation in the ion track, leads to decelerated convoy electrons $[11,12]$ and Auger electrons $[9,10,12]$. The corresponding positive potential attracts electrons and repels positively charged target ions [18], especially the light ones. One may estimate that a strong ion-track potential [9-12] existing for more than 10 fs will lead to significant atomic motion of light atoms, thereby suppressing any influence of hot electrons that return to the track at a too late stage of the evolution.

So far, experimental data have been published (except for a single spectrum for c-Si [20]) only for amorphous Si targets [19,16]. A small phase dependent peak-energy shift was found for $5 \mathrm{MeV} / \mathrm{u}$ Ar ions [20]. This first hint of a phase effect was the motivation for the present comprehensive study. The current work extends the previous data by results from 3 beamtimes for crystalline samples, one additional beamtime for amorphous $\mathrm{Si}$ and improved energy calibrations for all existing data. After a brief explanation of the 
experimental methods, electron and ion-induced electron spectra for $\mathrm{Si}$ as well as an analysis of the data are presented with special emphasis on phase effects in the ion-track potential observed for different Auger decay times.

\section{Experimental Method}

Experiments have been performed with fast highly charged particles at velocities between 6 and 10\% the speed of light (at 1.78 and $5 \mathrm{MeV} / \mathrm{u}$ ) delivered by the heavy-ion cyclotron of the IonenstrahlLabor (ISL) at the Hahn-Meitner-Institut Berlin. The setup is described in detail in ref.[19] and thus only a brief explanation shall be given here.

The heavy-ion beam of currents in the range of 100 to $500 \mathrm{nA}$ was focussed to a spot size of about $1.5 \times 1.5 \mathrm{~mm}$ at the target (normal incidence) inside a doubly magnetically shielded ultrahigh vacuum scattering chamber (working pressure slightly above $10^{-10}$ mbar, dominated by $\mathrm{H}_{2}$ ). Ion channeling for the parameter sets in this work is related to critical channeling half-angles of about $\pm 0.2^{\circ}$. The expected boundary angle of the focussed beam is about $\pm 0.4^{\circ}$ as estimated from slit-settings and the inner quadrupole-tube diameter and distance to the target. Typical misalignments of about $2^{\circ}$ served to suppress ion-channeling effects completely for the present ion velocity range.

A stripper foil in front of this chamber allows to choose a quasiequilibrium charge-state distribution. This method was applied for $1.78 \mathrm{MeV} / \mathrm{u} \mathrm{Xe}^{31+}$ ions, for $2.93 \mathrm{MeV} / \mathrm{u} \mathrm{Au}^{46+}$ ions as well as for $5 \mathrm{MeV} / \mathrm{u} \mathrm{Ne}^{9+}, \mathrm{Ar}^{16+}, \mathrm{Kr}^{29+}$ and $\mathrm{Xe}^{39+}$ ions. The corresponding average charge states have been estimated from ref. [16] and extracted from additional charge-state measurements for some of the cases. For $5 \mathrm{MeV} / \mathrm{u} \mathrm{Ar}^{16+}, \mathrm{Kr}^{29+}, \mathrm{Xe}^{39+}$ and for 1.78 $\mathrm{MeV} / \mathrm{u} \mathrm{Xe}^{31+}$ ions experiments have not only been performed with amorphous $\mathrm{Si}$, but also for the crystalline phase. Furthermore, we present data for the non-equilibrium charge-state ions 


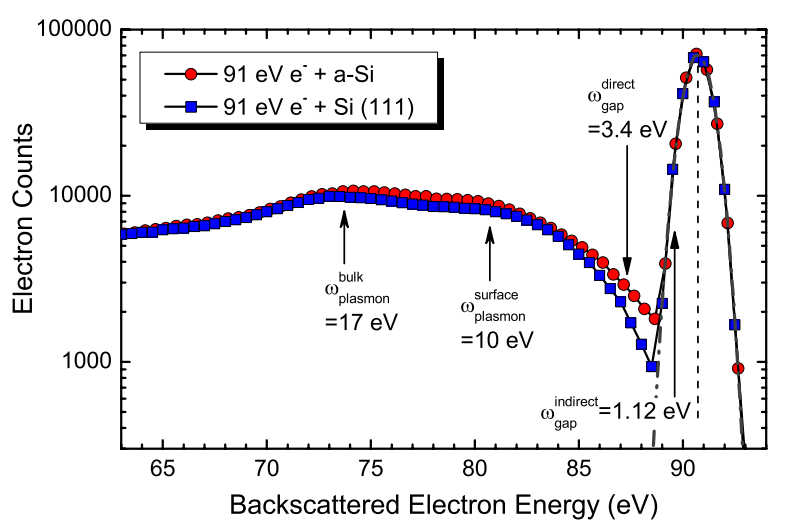

Fig. 1. (color online) Electron energy-loss spectra for electrons impinging on $\mathrm{Si}(111)$ $7 \times 7$ (blue squares) and for $\mathrm{Si}$ amorphized using Ar ions at about $4 \mathrm{keV}$ (red circles). Energy losses related to the direct and indirect gap as well as to surface and bulk plasmons are indicated by vertical arrows. The primary electron energy is about 91 $\mathrm{eV}$, the incident projectile-electron angle is $45^{\circ}$ with respect to the surface normal and the detected-electron direction lies in the same scattering plane at the all-over scattering angle of $90^{\circ}$.

$1.78 \mathrm{MeV} / \mathrm{u} \mathrm{Xe}^{15+}$ and $2.93 \mathrm{MeV} / \mathrm{u} \mathrm{Au}^{30+}$. In this case, we estimate that $<2$ projectile electrons will be stripped off within the first 3 surface layers (corresponding to the mean free path for the investigated Auger lines), leading to a slightly enhanced mean effective projectile charge-state.

As described in ref. [20] the surfaces of the boron doped $(<1 \Omega \mathrm{cm})$ $\mathrm{Si}(111)$ samples were chemically etched and sputter cleaned using 2.5 to $5 \mathrm{keV}$ Ar ions prior to the production of the crystalline phase. Target heating up to $850^{\circ} \mathrm{C}$ was performed for 20 to 40 minutes to crystallize respectively to recrystallize the sample and to form the $\operatorname{Si}(111) 7 \times 7$ reconstructed surface. The atomically clean target surfaces were characterized by electron spectroscopy and low-energy electron-diffraction (see our observed LEED pattern for the Si $7 \times 7$ surface in ref.[20]) before and after the ionirradiation cycles. During the scheduled cyclotron beam-times we used roughly $70 \%$ of the actual measurement time for incident ions (in order to reduce non-statistical fluctuations) and 30\% for incident electrons (distributed for reference purposes in between the ion runs). 


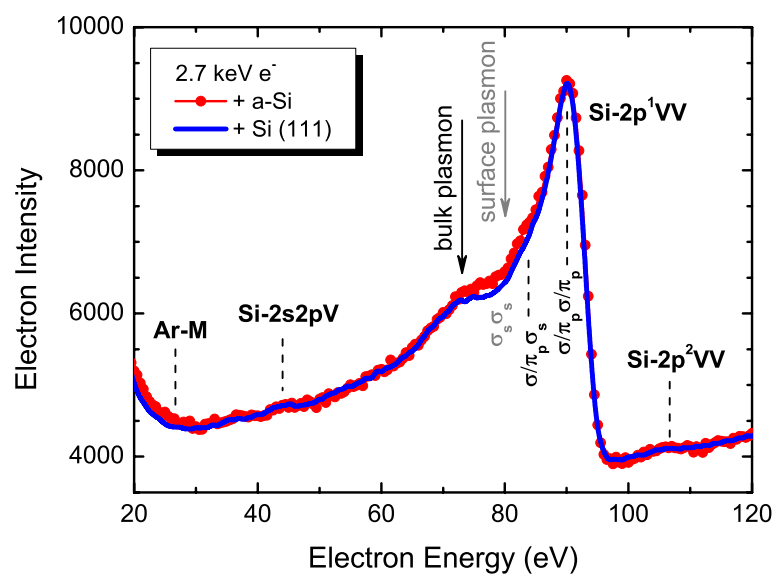

Fig. 2. (color online) Auger electron spectra normalized on the target current for $2.7 \mathrm{keV}$ electrons impinging on crystalline $\mathrm{Si}(111) 7 \times 7$ (solid blue curve) and on amorphized Si (red circles). Energy losses related to surface and bulk plasmons are indicated by vertical arrows. Band-structure contributions of the $S i-2 p^{1} V V$ as well as additional Si Auger lines $\left(S i-2 p^{2} V V, S i-2 s^{1} 2 p V\right)$ are marked with dashed vertical lines. The incident projectile-electron angle and the detected-electron direction are $45^{\circ}$ with respect to the surface normal, involving a difference in angles of $90^{\circ}$.

In some cases electron energy-loss spectra such as in Fig. 1 have been taken. As explained above, Auger-electron spectra as reference data sets have always been taken. An average over such spectra for one of the beam times is shown in Fig. 2. In this paper we present data for the crystalline as well as for the amorphous phase. Amorphized Si surface layers of a thickness of about $12 \mathrm{~nm}$ (range + straggling as obtained from the SRIM2003 code [21]) have been produced by irradiation with $5 \mathrm{keV} \mathrm{Ar}^{+}$ions for a few minutes. The amorphous structure was verified by the absence of any LEED spots before and after the measurement cycles. Furthermore, electron energy-loss spectra and electron induced Auger-electron spectra as in Figs. 1 and 2 have also been used to verify a stable Si phase. The Auger spectra, however, are only slightly sensitive to the electronic structure close to the band gap (with a small but significant intensity variation between 73 and $85 \mathrm{eV}$ in Fig. 2).

In order to avoid recrystallization of the amorphized samples due 
to the heat load by the fast heavy-ion beam, a special sample holder with improved cooling rates was used for the ion experiments. Dependent on the electrical power of the implanted heavyion beam, the reference temperature of the cooling system was either room temperature or liquid nitrogen temperature. For amorphized samples a narrow beam spot of about $1.5 \times 1.5 \mathrm{~mm}$ was used, in order to enhance the rates for nuclear amorphization. Contrary, for the crystalline samples the beam was defocussed to a few mm. Furthermore, the cooling system has been switched off in this case and high beam currents, leading to target temperatures of a few hundred degree $\mathrm{C}$, have been used for continuous recrystallization. In this paper we present only those results for which the target phase is well defined.

Electron spectra have been taken for heavy ions at normal incidence as well as for electrons of similar velocity (1, 2.7 and 7 $\mathrm{keV}$ ) at an incidence angle of $45^{\circ}$. During the experiments, the well-focussed electron beam is centered within the ion irradiated spot with an uncertainty of about $\pm 0.5 \mathrm{~mm}$ or better.

Since a precise reproducibility is an important ingredient in this work, we have analyzed the electron-energy calibration using the Al- $\mathrm{L}^{1} \mathrm{VV}$ Auger peak for a variety of experimental conditions. Contrary to what we have assumed before [20], we found signatures of a charging of the electron spectrometer that leads to a small positive energy shift for the detected electron energies. This shift was not observed before, since it is significant only for incident electrons at high energy when low-energy electrons are detected. This shift was showing a relatively good reproducibility. It was not observed for fast detected electrons $(>500 \mathrm{eV})$, not for primary electrons below $1 \mathrm{keV}$ and not for low currents or ions as projectiles. All evaluated electron-reference peak positions given in this paper are corrected (by 0.2 to $0.5 \mathrm{eV}$ ) for this effect. For most data points, this is a correction to within the error bars.

As has been shown in a previous work for carbon targets [22], Auger electrons ejected in backward directions are mainly in- 
duced directly by the projectile (in the central track region). Thus, electron spectra have been taken for a backward detection angle of $135^{\circ}$ with respect to the ion-beam direction, corresponding to $45^{\circ}$ with respect to the surface normal. Nearly all electron spectra have been taken with a relative energy resolution of $1.7 \%$. The most recent experimental data (for $2.93 \mathrm{MeV} / \mathrm{u} \mathrm{Au}^{30+/ 46+}$ and for $5 \mathrm{MeV} / \mathrm{u} \mathrm{Kr}^{29+}$ and $\mathrm{Xe}^{39+}$ ions), however, involve an energy resolution of $1 \%$ or even better. Ion and electron-induced Auger electron spectra obtained with these detection properties and with well-characterized Si surfaces are presented and analyzed in the next sections.

\section{Raw Data}

The electron induced Auger spectrum displayed in Fig. 3 has been taken for $7 \mathrm{keV}$ projectile electrons at the same scattering geometry as for the previous figures. The ion-induced target Auger spectrum of $\mathrm{Si}(111)$ in the same plot involves Auger structures at electron energies between 88 and $120 \mathrm{eV}$ due to one up to three $\mathrm{L}$-shell vacancies in the $2 \mathrm{p}$ shell $\left(2 \mathrm{p}^{1} \mathrm{VV}, 2 \mathrm{p}^{2} \mathrm{VV}\right.$, and $2 \mathrm{p}^{3} \mathrm{VV}$ ). Furthermore, a vacancy in the $2 \mathrm{~s}$ shell leads preferentially to the fast $2 \mathrm{~s} 2 \mathrm{p}^{m} \mathrm{~V}$ Koster-Kronig transitions (shown in Fig. 2). Compared to Fig. 2 the energy scale in Fig. 3 is significantly magnified, in order to allow for a comparison of the shapes and positions of the electron- and ion-induced spectra.

Already the raw spectra in Fig. 3 indicate that the ion induced spectra involve broadened and shifted Auger lines compared to the incident electron data. The experimental spectra that have been used for data evaluation are obtained after background subtraction and peak separation as described previously $[16,19,20]$. No phase effect is observed for the relative peak intensities. Furthermore, the electron temperature as it may be extracted from the Auger-line broadening relative to the electron-reference data is equal for amorphous and crystalline samples to within the ex- 


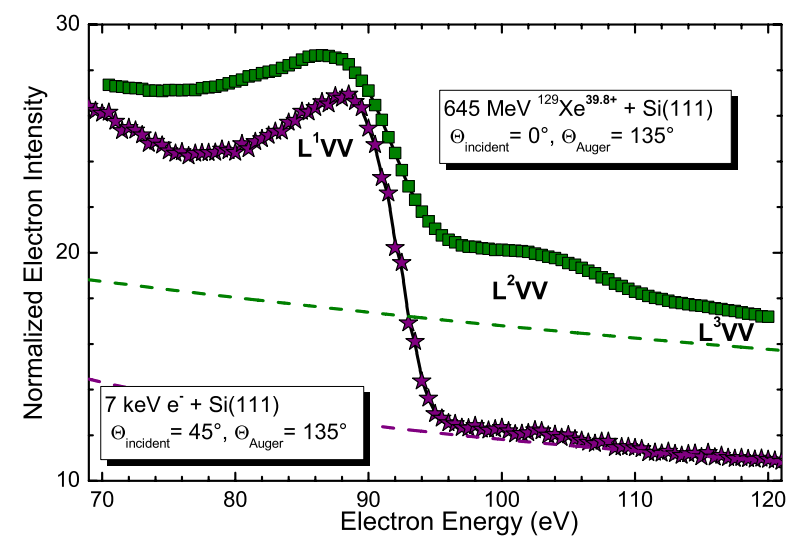

Fig. 3. (color online) Auger electron spectra (with arbitrary normalization) for $7 \mathrm{keV}$ electrons (purple stars, for a scattering geometry as in figures 1 and 2) impinging on a crystalline $\mathrm{Si}(111) 7 \times 7$ sample. For comparison an ion-induced spectrum (upper curve with green squares, for $645 \mathrm{MeV}{ }^{129} \mathrm{Xe}^{39+}$ ) for the same sample at normal incidence and at an detection angle of $135^{\circ}$ is shown together with fitted background functions (dashed curves) for both cases.

perimental uncertainties (no phase effect). Neglecting electroncollision cascades, it reaches about $\mathrm{T}_{e}\left(2 \mathrm{p}^{1} \mathrm{VV}\right)=15000 \mathrm{~K}$ for $\mathrm{Au}^{48+}$ ions when a simplified electronic density-of-states (eDOS) model is used for the semi-empirical fits (for details see ref. [13]). The same eDOS model indicates that there will be a small temperature drift for the Auger peak of $\Delta E_{\text {Auger }}\left(T_{e}\right)<0.05 \mathrm{eV}$. Thus, there is nearly no influence of the electron temperature on the Auger peak positions, which are discussed in the next section.

\section{Auger Peak-Energy Shifts}

In Fig. 4 the energy reduction of ion-induced Auger lines relative to the corresponding electron-induced spectra is displayed for a manyfold of Auger transitions, which correspond to different decay times. The results are displayed as function of the electronic perturbation parameter or interaction strength $\mathrm{P}=\left|q_{\text {eff }}\right| / v_{p}$, as it appears in quantum mechanical matrix elements for electronic excitations. The projectile velocity in units of the Bohr velocity $\left(2.19 \cdot 10^{6} \mathrm{~m} / \mathrm{s}\right)$ is denoted by $\mathrm{v}_{p}$, and the effective charge $\mathrm{q}_{\text {eff }}$ 


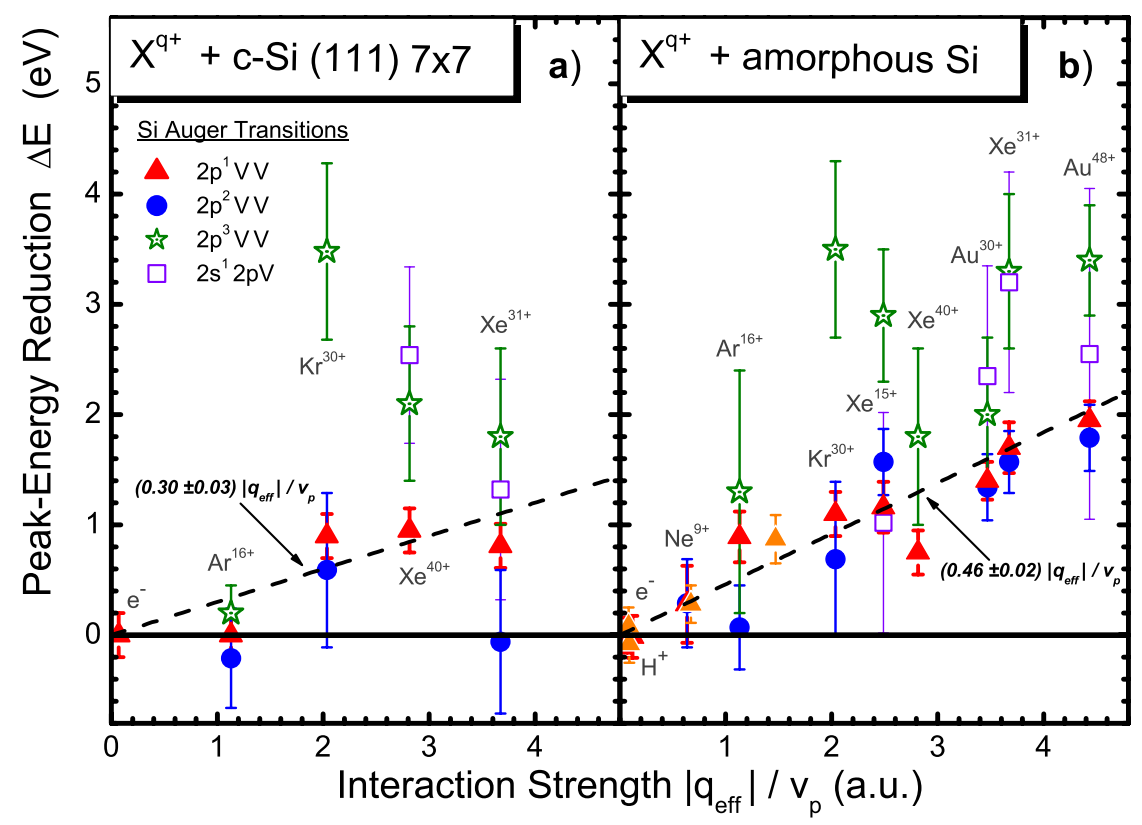

Fig. 4. (color online) Auger-peak energy reductions obtained from fits to spectra for amorphous Si (right-hand side, b) and for crystalline Si(111) 7x7 (left-hand side, a) excited by electrons at 1.0, 2.7 and $7 \mathrm{keV}$ and by different fast highly charged ions. The orange triangles in panel b) are obtained from an evaluation of the spectra from ref. [23]. The x-axis is given by the interaction strength $\mathrm{P}=q_{e f f} / v_{p}$.

is set equal to the mean particle charge state for most projectiles. Only for the non-equilibrium ions $1.78 \mathrm{MeV} / \mathrm{u} \mathrm{Xe}^{15+}\left(\mathrm{q}_{\text {eff }}\right.$ $=21)$ and for $0.94 \mathrm{MeV} / \mathrm{u} \mathrm{S}^{6+}\left(\mathrm{q}_{e f f}=9\right)$ [23], displayed in Fig. 4 , we have modified $\mathrm{q}_{\text {eff }}$ considering projectile electron-loss and reduced screening.

For the $2 \mathrm{~s} 2 \mathrm{pV}$ line, we have used the high-energy edge at $75 \%$ of the peak height for the corresponding energy determination in order to exclude a possible influence of multiple 2 p-ionization. For all other lines, the upper $15 \%$ of the peak have been considered. Except for the $2 \mathrm{p}^{3} \mathrm{VV}$ transition, where an extrapolated zero-shift value is used, we have taken data for incident electrons (after applying the small charging-up correction discussed in section 2) as reference data. Furthermore, we have reanalyzed the spectra for primary electrons, protons, $2 \mathrm{MeV} / \mathrm{u} \mathrm{O}^{6+}$, and $0.94 \mathrm{MeV} / \mathrm{u}$ $\mathrm{S}^{6+}$ on a-Si from the pioneering work by Schmidt et al. [23]. It is 
noted that the results by Koyama et al. [24] had to be excluded, since no electron reference-data have been published. The error bars in Fig. 4 indicate all-over estimates of the uncertainty. It is noted that we have not performed a correction for the influence of $\delta$-electron cascades on the $2 \mathrm{pVV}$ line position (this awaits a more detailed understanding of this line shape for $\mathrm{Si}$ ).

To within the experimental uncertainties, the peak shifts in Fig. 4 increase monotonically with the interaction strength $\mathrm{P}$ and reach values of 2 to $3 \mathrm{eV}$ at $\mathrm{P}=4.4$. For each transition the $\mathrm{P}$ dependence is statistically consistent with a linear curve through the origin. Since the corresponding slopes do vary only a little for the different types of transitions, we have plotted general leastsquare fit functions separately for amorphous and for crystalline $\mathrm{Si}$ as dashed lines in Figs. 4 a) and b). Macroscopic charging of the B-doped Si samples can be excluded as an explanation for the observed effect, since no indication of a target dependent peak shift could be found for incident electrons at different beam currents. Materials modification can be excluded as well, as follows from the observed consistent LEED structures.

In Fig. 5 specific peak-energy reductions are displayed as function of the mean Auger-decay time. The specific reductions are given by the slope of linear fits to the data in Fig. 4, performed separately for each Auger transition and for the two solid-state phases. The 1s-level width $(0.48 \mathrm{eV})$ and the 2 s-level width $(1.03$ $\mathrm{eV}$, corresponding to a decay time of 0.64 fs for a $2 \mathrm{~s} 2 \mathrm{pV}$ KosterKronig decay) are taken from ref. [25]. For a single $2 p$ vacancy $\left(2 p^{1}\right)$ we have performed a weighted average over the five lowest of recent level-widths measurements [26-30]. The resulting level width is $0.048 \pm 0.022 \mathrm{eV}$ corresponding to a decay time of 13.7 fs. These level-widths data are relatively uncertain, since the line widths are too small to be measured with high precision. The data for double $2 \mathrm{p}$-vacancies (3.25 fs) and for triple $2 \mathrm{p}$-vacancies (1.19 fs) result from a theoretical extrapolation of the experimental single-vacancy value. For this purpose, we have used numerical Hartree-Fock-Slater wavefunctions, renormalized to the Wigner- 


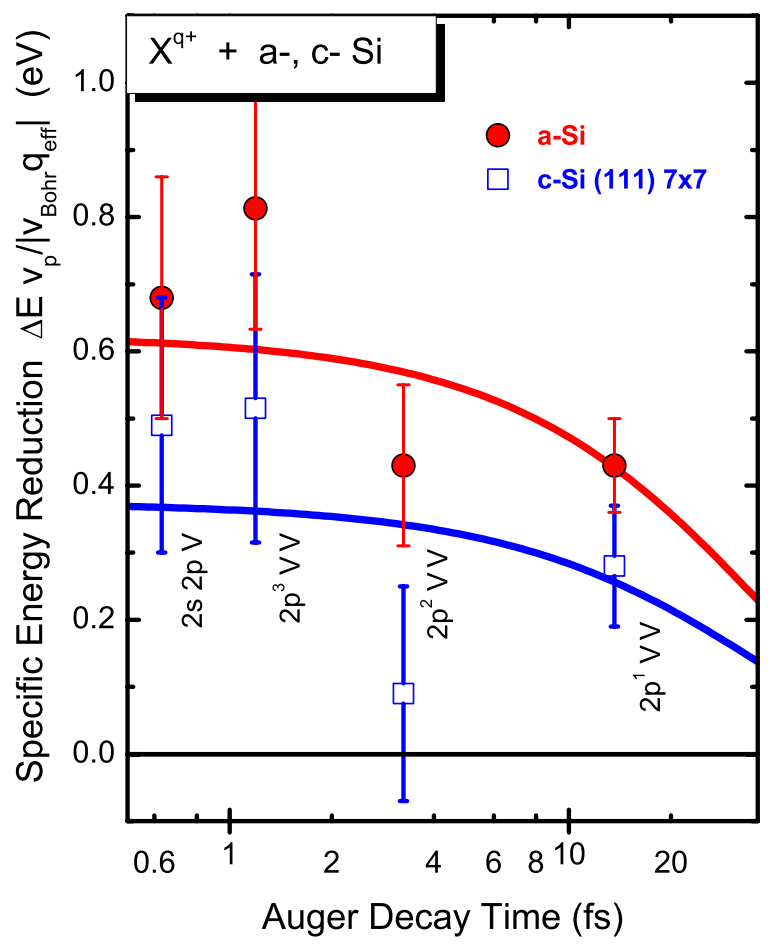

Fig. 5. (color online) Specific Auger-energy reductions obtained from linear fits to the data points in the previous figure for amorphous Si (closed red circles) and for crystalline $\mathrm{Si}(111) 7 \times 7$ (open blue squares) excited by different projectiles. The data are presented as function of the Auger-decay time for different Auger transitions.

Seitz volume and calculated the Auger matrix-elements assuming a local decay.

Observation of Fig. 5 shows that the specific shifts are similar for the different Auger lines. In other words, there is only a week time dependence of the shifts. A phase effect, namely a lower energy shift for c-Si compared to a-Si, is clearly visible in the figure. This phase effect, however, is less pronounced than expected on the basis of our first data set for crystalline Si [20].

We attribute the measured shift in Fig. 4 to the nuclear-track potential induced by a reduced electron density in the center of the track. Auger electrons are decelerated when they leave such a charged region. From our previous measurements of this effect for polypropylene and mylar $[9,10]$, we estimate that the initial track potential directly after the interaction with $\mathrm{Si}$ will exceed 
$100 \mathrm{~V}$ for the heaviest ions. Thus, the measured shift of only 2 to $3 \mathrm{eV}$ points to a rapid electronic neutralization of the track. This is also expected considering the high mobilities of electrons in the conduction band or holes in the valence band of Si. The remaining measured shift seems to be related to a very slow component that might be due to long-lived traps for electrons and for holes as well. More likely, it might be related to self-trapped excitons in the amorphous material. For the crystalline Si, thermally displaced atoms might enhance self-trapping. It should be noted, that the high surface-sensitivity of our Auger transitions may also magnify the influence of populated surface states on the peak position. In any case, these states have to be long-lived ( $>30 \mathrm{fs}$, as it may be extracted from Fig. 5) and trapped near the ion-track center.

\section{Conclusions}

In this work we have presented and analyzed Auger electron spectra induced by incident electrons and swift heavy ions in the $\mathrm{MeV} / \mathrm{u}$ regime. The different investigated Auger lines correspond to snapshots of the track evolution for times between $0.6 \cdot 10^{-15} \mathrm{~s}$ (decay of the $2 \mathrm{~s}$ vacancy) and $14 \cdot 10^{-15} \mathrm{~s}$ (2p Auger decay). The target surfaces, either amorphous $\mathrm{Si}$ or $\mathrm{Si}(111)$ with $7 \times 7$ reconstruction, were characterized by Auger-electron spectroscopy and low-energy electron diffraction (LEED) before and after the ion-irradiation runs. Off-line electron energy-loss spectroscopy (EELS) has also been applied for the same samples. The present data allow for the first time to compare two Si phases quantitatively regarding their short-time electron dynamics.

All investigated Auger peak positions show a small but significant shift towards lower energy when the charge of the projectile is increased. Transport calculations considering the electronic density-of-states show that the peak positions should not be influenced by the electron temperature in the track. This finding is consistent with a slow and weak component of the nuclear- 
track potential at the center of the ion track. Comparison of the Auger electron spectra for amorphous and crystalline Si indicates a phase effect in the short-time dynamics of ion tracks, involving the nuclear-track potential, but not the electron temperature extracted from the data. Charge neutralization seems to be suppressed by $50 \%$ in amorphous Si compared to crystalline Si targets, leading to enhanced deceleration of Auger electrons.

One may speculate that displaced atoms resulting from the atomic structure or from the thermal atomic motion may increase the number of self-trapped excitons. The corresponding reduced allover electron density would then give rise to a small nucleartrack potential. A nuclear-track potential of up to $3 \mathrm{eV}$ existing for about $30 \mathrm{fs}$, however, might give rise to an energy transfer to the lattice that is comparable to the one resulting from hot electrons at electron temperatures of 10000 to $20000 \mathrm{~K}$ in Si. Thus, Si might be a material where the nuclear-track potential and the electron temperature are both important. As expected, however, our auxiliary LEED investigations are consistent with amorphization by the nuclear energy loss only. Thus, we conclude that none of our heavy-ion energy-losses was significantly above the electronic energy-loss threshold for surface amorphization in Si.

Nevertheless, the present results for the nuclear-track potential might have a significant impact on the understanding of the socalled pulse-height defect (PHD) in a charged-particle Si detector [31-38]. This PHD is related to a non-proportionality between the deposited ion energy and the measured total charge in the detector and it is due to

- nuclear energy transfers in the Si crystal that do not lead to charge-carrier production

- a (usually small) amount of ion energy-losses in non-sensitive zones (the $\mathrm{Au}$ entrance window of the detector and a possible dead layer underneath)

- a residual plasma defect of up to about $10 \%$ related to high 
ionization densities and charge-carrier recombination

The residual plasma defect is found for fast heavy ions [31-34] or for cluster projectiles [35] as long as the cluster constituents are moving close together. It depends on the electric field inside the detector (around $10 \mathrm{kV} / \mathrm{cm}$, determined by the detector biasvoltage) [36-38] and it influences even the measured pulse shapes [38]. The current understanding suggests [38] that the chargecarrier transport is suppressed near the ion tracks, because the external electric field cannot penetrate the conducting electronhole plasma. Thus, charge carriers may recombine before reaching an electrode.

The density of the electron-hole plasma in the track is increasing with the electron temperature as it shows up also in this investigation. However, the nuclear-track potential is related to strong electric fields and maybe also to field fluctuations orders of magnitude above typical external field strengths. Hence, these internal ion-track fields should be considered in addition to the external ones for the pulse shape and PHD in Si solid-state detectors. With the present investigation, however, we are sensitive to time scales that are 6 orders of magnitude shorter than the pulseshape effects. Thus, the behavior of solid-state detectors should be critically dependent on the relaxation dynamics of the electron temperature and of the nuclear-track fields. Furthermore, the well-known pulse-height reduction due to degradation of Si surface-barrier detectors might partly be related to an amplification of the phase effect observed ion this work.

\section{Acknowledgments}

We acknowledge the support by the Alexander-von-Humboldt foundation and by PROBRAL (DAAD/Capes contract). We would also like to acknowledge the very helpful comments by and discussions with R.Baragiola and S.Klaumünzer. 
+ Present address: Inst. of Physics, University of Szczecin, ul. Wielkopolska 15, 70-451 Szczecin, Poland.

++ Present address: Forschungszentrum Dresden-Rossendorf, Bautzner Landstr. 128, 01328 Dresden, Germany.

\section{References}

[1] P.L. Grande and G. Schiwietz, "Ionization and energy loss beyond perturbation theory ", in "Advances in Quantum Chemistry", vol. 45, pp.7-46 (book article ed. by J. Sabin, 2004, Elsevier Inc.).

[2] R.L. Fleischer, P.B. Price, and R.M. Walker, "Nuclear Tracks in Solids" (University of California Press, Berkely California, 1975).

[3] R. Spohr, "Ion tracks and microtechnology" (F.Vieweg und Sohn Verlagsgesellschaft, Braunschweig 1990).

[4] P. Stampfli, K.H. Bennemann, Phys. Rev. B49, 7299 (1994); P. Stampfli, Nucl. Instr. Meth. B107, 138 (1996).

[5] Z.G. Wang, C. Dufour, E. Paumier and M. Toulemonde, J. Phys.: Condens. Matter 6, 6733 (1994).

[6] A.E. Volkov, V.A. Borodin, Nucl. Instr. Meth. B107, 172 (1996).

[7] S.Klaumünzer, Ming-dong Hou and G.Schumacher, Phys.Rev.Lett. 57, 850 (1986).

[8] S.Klaumünzer, Matematisk-fysiske Meddelelser 52, 293-328 (2006).

[9] G. Schiwietz, P.L. Grande, B. Skogvall, J.P. Biersack, R. Köhrbrück, K. Sommer, A. Schmoldt, P. Goppelt, I. Kádár, S. Ricz, and U. Stettner, Phys. Rev. Lett. 69, 628 (1992).

[10] G. Schiwietz, G. Xiao, Nucl. Instr. Meth. B107, 113 (1996).

[11] G. Xiao, G. Schiwietz, P.L. Grande, A. Schmoldt, N. Stolterfoht, M. Grether, R. Köhrbrück, A. Spieler and U. Stettner, Phys. Rev. Lett. 79, 1821 (1997).

[12] G. Schiwietz, E. Luderer, G. Xiao and P.L. Grande, Nucl. Instr. Meth. B175177, 1-11 (2001).

[13] G. Schiwietz, G. Xiao, P.L. Grande, E. Luderer, R. Pazirandeh, U. Stettner, Nucl. Instr. Meth. B146, 131-136 (1998); - ibid, Europhys. Lett. 47, 384-390 (1999), G. Schiwietz, G. Xiao, E. Luderer, and P.L. Grande, Nucl. Instr. Meth. B164, 353-364 (2000).

[14] M. Caron, H. Rothard, M. Beuve, B. Gervais, Physica Scipta T92, 281 (2001). 
[15] F. Staufenbiel, G. Schiwietz, K. Czerski, M. Roth, and P.L. Grande, Nucl. Instr. Meth. B230, 426-430 (2005).

[16] G. Schiwietz, M. Roth, K. Czerski, F. Staufenbiel, and P.L. Grande, Nucl. Instr. Meth. B225, 4-26 (2004) and Nucl. Instr. Meth. B226 (2004) 683-704.

[17] G. Schiwietz, M. Roth, K. Czerski, F. Staufenbiel, and P.L. Grande, Phys. Rev. Lett. 99, 197602-1 -4 (2007).

[18] K. Wien, Ch. Koch, and Nguyen van Tan, Nucl. Instr. Meth. B100, 322 (1995).

[19] G. Schiwietz, K. Czerski, M. Roth, F. Staufenbiel, E. Luderer, P. L Grande, Nucl. Instr. Meth. B193, 705-712 (2002), the 2pVV surface-plasmon assignment in this paper is false, as follows from a more detailed analysis.

[20] G. Schiwietz, M. Roth, K. Czerski, F. Staufenbiel, M. Rösler, and P.L. Grande, Nucl. Instr. Meth. B209, 26-31 (2003).

[21] semi-empirical range prediction from the SRIM2003 code (version 20) by J. F. Ziegler and J.P. Biersack.

[22] G. Schiwietz, D. Schneider, J.P. Biersack, N. Stolterfoht, D. Fink, A. Mattis, B. Skogvall, H. Altevogt, V. Montemayor, U. Stettner, Phys. Rev. Lett. 61, 2677 (1988).

[23] W. Schmidt, P. Müller, V. Brückner, F. Löffler, G. Saemann-Ischenko, W. Schubert, Phys. Rev. A24, 2420 (1981).

[24] A. Koyama, H. Ishikawa, K. Maeda, Y. Sasa, O. Benka, M. Uda, Nucl. Instr. Meth. B48, 608 (1990).

[25] M. Krause, J. Phys. Chem. Ref. Data 8, 507 (1979).

[26] K. Hricovini, R. Günther, P. Thiry, A. Taleb-Ibrahimi, G. Indlekofer, J.E. Bonnet, P. Dumas, Y. Petroff, X. Blase, X. Zhu, S.G. Louie, Y.J. Chabal, and P.A. Thiry, Phys. Rev. Lett. 70, 1992-1995 (1993).

[27] R. I. G. Uhrberg, T. Kaurila, and Y.-C. Chao, Physical Review B58, R1730R1733 (1998).

[28] K.S. An, C.C. Hwang, Y.K. Kim, E.S. Cho, C.-Y. Park, S. Pukird, A. Kakizaki, T. Okuda, T. Kinoshita, Surface Science 513, 49-56 (2002).

[29] R.D. Bringans, M.A. Olmstead, R.I.G. Uhrberg, and R.Z. Bachrach, Physical Review B36, 9569-9580 (1987).

[30] X. Tong, S. Ohuchi, T. Tanikawa, A. Harasawa, T. Okuda, Y. Aoyagi, T. Kinoshita, S. Hasegawa, Applied Surface Science 190, 121-128 (2002).

[31] H.C. Britt and H. E. Wegner, Rev. Sci. Instr. 34, 274 (1963).

[32] F. M. Ipavich, R. A. Lundgren, B. A. Lambird and G. Gloeckler, NIM154, 291-294 (1978). 
[33] J. B. Moulton, J. E. Stephenson+, R. P. Schmitt and G. J. Wozniak, NIM157, 325-331 (1978).

[34] G. Pasquali, G. Casini, M. Bini, S. Calamai, A. Olmi, G. Poggi, A.A. Stefanini, F. Saint-Laurent, J.C. Steckmeyer, NIM-A405, 39-44 (1998).

[35] M. Seidl, H. Voit, S. Bouneau, A. Brunelle, S. Della-Negra, J. Depauw, D. Jacquet, Y. Le Beyec, and M. Pautrat, NIM-B183, 502-508 (2001).

[36] E. C. Finch, M. Asghar, M. Forte, NIM163, 467-477 (1979).

[37] M. Ogihara, Y. Nagashima, W. Galster and T. Mikumo, NIM-A251, 313 (1986).

[38] G. Pausch, W. Bohne and D. Hilscher, NIM-A337, 573-587 (1994). 